\title{
EIN ANALOGON ZUM HIGH INDICES THEOREM FÜR POTENZREIHEN MIT WENIGEN VORZEICHENWECHSELN
}

BY JOACHIM KÜHN

Communicated by Maurice Heins, August 21, 1967

1. Problem und Ergebnis. Es sei

$$
f(z)=\sum_{1}^{\infty} a_{n} z^{n}
$$

eine in $\{z|| z \mid<1\}$ konvergente Potenzreihe mit reellen Koeffizienten. Man sagt, daß der Koeffizient $a_{m}$ einen Vorzeichenwechsel bestimmt, wenn erstens $a_{m} \neq 0$ ist und zweitens das Vorzeichen von $a_{m}$ dem des letzten $a_{m}$ vorangehenden nichtverschwindenden Koeffzienten entgegengesetzt ist. Mit

$$
\left\{\nu_{k}\right\}
$$

wollen wir die Folge der Indizes derjenigen Koeffizienten von (1) bezeichnen, die einen Vorzeichenwechsel bestimmen. Wir setzen voraus, daß die Folge (2) eine $q$-Hadamardfolge ist, d.h. es gibt ein $q>1$, so daß

$$
\nu_{k+1} / \nu_{k} \geqq q \quad(k=1,2, \cdots) .
$$

Für solche Potenzreihen gilt der folgende, zum High Indices Theorem von Hardy-Littlewood (etwa [4], S.173) Analoge

Satz. (i) Die Folge $\left\{\nu_{k}\right\}$ sei eine $q$-Hadamardfolge, und es sei $|f(x)|$ $\leqq A \quad(0 \leqq x<1)$. Dann gilt $\left|a_{n}\right| \leqq K A n$, wobei die Konstante $K$ nur von $q$ abhängt.

(ii) Unter den genannten Voraussetzungen ist die Größenordnung $a_{n}=O(n)$ bestmöglich.

Wir benutzen zum Beweis ähnliche Methoden wie Edrei [1], Gaier [2] und Halász [3]. Genauere Ausführungen zu diesem Satz sowie verwandte Sätze werden in der Arbeit [5] des Verfassers behandelt.

2. Hilfsmittel. Im folgenden sei $D$ die längs der negativ reellen Achse geschlitzte Ebene. Von der Wurzelfunktion wollen wir stets denjenigen $Z$ weig nehmen, der in $D$ regulär ist und durch $\sqrt{1}=1$ bestimmt ist.

Für die Folge (2) bilden wir mit $\omega_{k}=\nu_{k}-\frac{1}{2}$ das Blaschkeprodukt 


$$
B(z)=\prod_{1}^{\infty} \frac{\sqrt{ } \omega_{k}-\sqrt{ } z}{\sqrt{ } \omega_{k}+\sqrt{ } z}=\prod_{1}^{\infty}\left(1-\frac{2 \sqrt{ } z}{\sqrt{ } \omega_{k}+\sqrt{ } z}\right),
$$

das wegen (3) in $D$ konvergiert.

Hilfssatz 1. Fïr das Blaschkeprodukt (4) gilt $|B(n)|^{-1} \leqq C n$ $(n=1,2, \cdots)$, wobei die Konstante $C$ nur von $q$ abhängt.

Es sei $\left\{\lambda_{n}\right\}$ eine Folge reeller Zahlen mit $\lambda_{n} \in\langle n, n+1\rangle$ $(n=1,2, \cdots)$ und $\mu_{n}=n^{2} / \lambda_{n}$ gesetzt. Wir bilden die in $D$ meromorphe Funktion

$$
G(z)=\prod_{1}^{\infty} \frac{1-z / n}{\left(1+\left(z / \mu_{n}\right)^{1 / 2}\right)\left(1-\left(z / \lambda_{n}\right)^{1 / 2}\right)}
$$

mit einfachen Nullstellen an $z=n$ und einfachen Polen an $z=\lambda_{n}$ $(n=1,2, \cdots)$.

Hilfssatz 2. Die Funktion $G$ hat die folgenden Eigenschaften:

(a) auf dem Rand von $D$ gilt $|G(z)| \leqq 1$,

(b) auf Kreisen $\{z|| z \mid=R\}$ mit $R>1$ und $\left|R-\lambda_{n}\right|>\frac{1}{4}$ $(n=1,2, \cdots)$ gilt $|G(z)| \leqq K|z|^{4}$, wobei die Konstante $K$ nicht von $\left\{\lambda_{n}\right\}$ abhängt,

(c) $|\sin \pi x / G(x)| \leqq 32(x+1) \quad(x>1)$.

3. Beweisskizze. Für ein festes $\delta \in(0,1)$ bilden wir $f_{\delta}(z)=f(\delta z)$ $=\sum_{1}^{\infty} a_{n} \delta^{n} z^{n}$ und studieren die Hilfsfunktion

$$
H_{\delta}(z)=\int_{0}^{1} f_{\delta}(t) t^{-z-1} d t \quad(z=x+i y) .
$$

Da $f_{\delta}(t) / t$ im Intervall $\langle 0,1\rangle$ regulär ist, ist $H_{\delta}$ in $\{z \mid x<1\}$ definiert und stellt dort eine reguläre Funktion dar. Auf der negativ reellen Achse gilt

$$
\left|H_{\delta}(-x)\right| \leqq A / x \quad(x>0) .
$$

Durch gliedweise Integration erhält man für $\operatorname{Re} z<0$

$$
H_{\delta}(z)=\sum_{1}^{\infty} \frac{a_{n}}{n-z} \delta^{n}
$$

und erkennt daran, daß $H_{\delta}$ eine in der ganzen Ebene meromorphe Funktion mit einfachen Polen an $z=n$ und den dazugehörigen Residuen $-a_{n} \delta^{n}$ ist. Folglich ist die Funktion

$$
\Phi_{\delta}(z)=H_{\delta}(z) \sin \pi z
$$


eine ganze Funktion, die auf der reellen Achse nur reelle Werte annimmt.

Wir interessieren uns für die positiven Nullstellen dieser Funktion. Dazu benötigen wir die Eigenschaft

$$
\Phi_{\delta}(n)=(-1)^{n+1} a_{n} \delta^{n} \pi \quad(n=1,2, \cdots) .
$$

Wir diskutieren den einfachsten Fall $a_{n} \neq 0 \quad(n=1,2, \cdots)$. Gilt $a_{n} a_{n+1}>0$, so liegt wegen (9) im Intervall $(n, n+1)$ mindestens eine Nullstelle $\lambda_{n}$ von $\Phi_{\delta}$; gilt $a_{n} a_{n+1}<0$, d.h. bestimmt $a_{n+1}$ einen Vorzeichenwechsel, so braucht im Intervall $(n, n+1)$ keine Nullstelle zu liegen. Die Nullstellen fehlen also höchstens in den Intervallen $\left(\nu_{k}-1, \nu_{k}\right)$. Um auch dort Nullstellen zu bekommen, bilden wir mit dem Blaschkeprodukt (4) $\Psi_{\delta}(z)=\Phi_{\delta}(z) B(z)$. Diese Funktion hat nun in jedem Intervall $(n, n+1)(n=1,2, \cdots)$ mindestens eine Nullstelle $\lambda_{n}$.

Mit $\mu_{n}=n^{2} / \lambda_{n}$ betrachten wir

$$
\begin{aligned}
F_{\delta}(z)=\frac{\Psi_{\delta}(z)}{\pi} & {\left[\prod_{1}^{\infty}(1+z / n) e^{-z / n}\right.} \\
& \left.\prod_{1}^{\infty}\left(1+\left(z / \mu_{n}\right)^{1 / 2}\right) \cdot\left(1-\left(z / \lambda_{n}\right)^{1 / 2}\right) e^{z / n}\right]^{-1}(z \in D)
\end{aligned}
$$

und stellen Eigenschaften von $F_{\delta}$ zusammen:

(a) $F_{\delta}$ ist in $D$ regulär,

(b) auf dem Rand von $D$ gilt wegen (7) und wegen des Hilfssatzes 2 $\left|F_{\delta}(z)\right| \leqq A$,

(c) es gibt eine Folge von Kreisen $\left\{z|| z \mid=R_{\nu}\right\} \quad\left(R_{\nu} \rightarrow \infty\right)$, auf welchen $F_{\delta}(z)=O\left(|z|^{5}\right) \quad(\nu \rightarrow \infty)$ gilt. Wegen des Pragmén-Lindelöfschen Prinzips erhalten wir

$$
\left|F_{\delta}(z)\right| \leqq A \quad(z \in D) .
$$

$\mathrm{Da}$

$$
\begin{aligned}
& \left|F_{\delta}(n)\right|=\left|a_{n}\right| \delta^{n} \pi|B(n)| \\
& \cdot\left[\prod_{m=1}^{\infty}\left(1+\left(n / \mu_{m}\right)^{1 / 2}\right)\left|1-\left(n / \lambda_{m}\right)^{1 / 2}\right| e^{n / m} \pi \prod_{m=1}^{\infty}(1+n / m) e^{-n / m}\right]^{-1},
\end{aligned}
$$

folgt wegen der Hilfssätze 1 und 2

$$
\left|a_{n}\right| \delta^{n} \leqq 32 C A n(n+1) / n \pi .
$$

Läßt man $\delta \rightarrow 1$ streben, so ergibt sich die Behauptung (i).

Die Behauptung (ii) belegen wir durch das Beispiel 


$$
f(z)=\sum_{k=0}^{\infty}(-1)^{k} 2^{k}\left(z^{2 k}=z^{2 k+1}\right) .
$$

Diese Reihe konvergiert im Einheitskreis, die Folge der Indizes derjenigen Koeffizienten, die Vorzeichenwechsel bestimmen, ist $\left\{2^{k-1}+1\right\}$, also ersichtlich eine Hadamardfolge, und die Koeffizienten bei $z^{2^{k}}$ sind $\pm 2^{k}$. Durch leichte Rechnung zeigt man $f(x)=O(1)$ $(x \rightarrow 1)$.

\section{LITERATUR}

1. A. Edrei, Gap and density theorems for entire functions, Scripta Math. 23 (1957)' 117-141.

2. D. Gaier, On the coefficients and the growth of gap power series, SIAM J. Numer. Anal. 3 (1966), 248-265.

3. G. Halász, Some complementary remarks to a paper of Mr. Gaier on gap theorems, Acta Sci. Math. (Szeged).

4. G. H. Hardy, Divergent series, Oxford Univ. Press, New York, 1949.

5. J. Kühn, Über das Wachstum reeller Potenzreihen mit wenigen Vorzeichenwechseln und iiber das Wachstum ganzer Dirichlet-Reihen, Mitt. Math. Sem. Giessen 75 (1967).

UnIVERSität GiesseN 